affected the velocity of its light, in would affect the time required for the light to reach us and would, therefore, change the apparent position of the star at any instant. The resulting distortion, which in some cases would be great, has been carefully looked for but has been found absent.

Thus it is a fact founded on observation. that the velocity of light is independent of the source. The meaning of this fact can be made clear in the following way. Suppose an observer imprisoned in a windowless box which is thrown at random into space. Such an observer, meeting no experimental diffculties, could discover and accurately define his speed through space by simply measuring the velocity of light in various directions within his enclosure, and this, be it noted, without any reference whatever to any outside body. In general, he would find that light travels faster across his box in one direction. than it does in another, for, as we have seen, the real velocity of light in space is not affected by the motion of the light source which he carries, and hence a change in his. motion would change the apparent velocity of light within his box.

Thus space possesses what we might call a 'positional' property, by means of which the magnitude of any motion can be defined without reference to any body in the universe, and this motion is what physicists call 'motion with respect to the ether.'

There is an entirely different experimental truth which leads to the same conclusion as. the above.

It is a generally accepted truth that two similarly charged bodies when moved side by side have, superimposed upon their mutual repulsion, an attraction which depends upon the fact that when moving they act like two parallel electric currents. This follows from Roland's classical experiment.

Now if the two charges, stationary with respect to each other, are considered alone in space, it is evident that they furnish our imprisoned experimenter another means of finding his motion relative to space, for the strength of the above-mentioned attraction depends only upon this aksolute motion. This leads to the same conclusion as formerly, that space has a 'positional' property.

Other evidence of a similar kind might be given, but the above is sufficient to make it clear that at least so far as it represents this positional property of space the conception of an ether is thoroughly legitimate.

A being from a planet which possessed no atmosphere, if he came to earth, might first become conscious of our atmosphere through feeling it set in motion relatively to himself when he moved. In a somewhat similar way the ether manifests itself, since we know it through its motional property.

We are conscious of matter only as a collection of properties and one of these properties certainly is that it is capable of marking position. Therefore, the unknown reality, which exhibits this positional attribute in space as one of its properties, can be said to resemble matter to this limited extent at least, and upon this sure foundation can fitly be based the physicist's conception of an ether.

Daniel F. Comstock

Cavendish Laboratory, CAMBRIDGE

\section{NOTES ON ORGANIC CHEMISTRY}

GLYOXAL

THE properties of the substance known as glyoxal, which is the simplest dialdehyde, certainly do not accord with its formula, OCHCHO. Some text-books try to evade this difficulty by describing it as 'a hydrate,' but this, to say the least, is hardly satisfactory. The matter has been cleared up very completely by a recent paper of Harries and Temme. ${ }^{1}$ Glyoxal is found to exist in four forms : (1) Monomolecular glyoxal, OCHCHO, which is unstable and is described more fully below. (2) A trimolecular modification $\left(\mathrm{C}_{2} \mathrm{H}_{2} \mathrm{O}_{2}\right)_{3}$, which is a colorless, pulverulent solid, readily soluble in water. It forms no characteristic derivatives, $i$. e., reagents give with it only derivatives of (1). (3) Paraglyoxal $\left(\mathrm{C}_{2} \mathrm{H}_{2} \mathrm{O}_{2}\right)_{x}$, a white powder, insoluble in water. (4) The ordinary form of glyoxal, now termed polyglyoxal $\left(\mathrm{C}_{2} \mathrm{H}_{2} \mathrm{O}_{2}\right)_{n}$, which has been known during the past fifty years.

${ }^{1}$ Ber. d. Chem. Ges., 40, 165 (1907). 
Monomolecular glyoxal is prepared by distilling a mixture of commercial glyoxal (4) with phosphorus pentoxide, and cooling the distillate by means of solid carbon dioxide and ether. Care must be taken to avoid the presence of moisture in the apparatus. The compound crystallizes in yellow prisms or spangles, becomes opaque at $10^{\circ}$, melts at $15^{\circ}$ and boils at $50^{\circ}$. The vapor is intensely emerald green and condenses to a liquid which is at first green; as this is cooled it becomes yellow and, at very low temperatures, colorless. The vapor has an odor like that of formic aldehyde, but as inhalation continues the smell becomes sweet and not disagreeable. It burns with a violet flame and forms with air a mixture which explodes violently on the application of a flame. Glyoxal changes spontaneously into paraglyoxal (3) in a few hours, but on the addition of a little water the transformation is instantaneous. If, however, glyoxal be added to a large volume of water it dissolves with a hissing noise and the resulting liquid, which has a decidedly acid reaction, consists of an aqueous solution of monomolecular glyoxal (1). The glyoxal volatilizes with the steam when the solution is boiled. The solution readily reduces ammoniacal silver nitrate solution, but not Fehling's solution, in which latter respect it resembles the poly- and para-modifications.

Trimolecular glyoxal (2) is readily prepared by treating cinnamic aldehyde, $\mathrm{O}_{6} \mathrm{H}_{6} \mathrm{CH}$ : CHCHO, with ozone. The resulting ozonide, when mixed with water, gives hydrogen peroxide, benzoic aldehyde (or benzoic acid) and the glyoxal. The reaction may be represented by the formula,

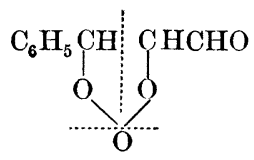

the dotted lines showing the positions at which cleavage occurs.

The intense color of glyoxal is very interesting, the simplest diketone, diacetyl, $\mathrm{CH}_{3} \mathrm{COCOCH}_{3}$, is also strongly colored, whereas oxalic acid, HOCOCOOH, is absolutely colorless, yet all three substances possess in common the grouping OCCO, in one case united with hydrogen or methyl (colored), in the other combined with hydroxyl (colorless). It is tolerably certain that, of the compounds consisting only of the elements carbon, hydrogen and oxygen, glyoxal is the simplest one to exhibit color.

\section{Johns Hopkins University}

J. Bishop Tingle

\section{CURRENT NOTES ON METEOROLOGY AND CLIMATOLOGY}

HAIL-SHOOTING-THE QUESTION SETTLED

IT may be remembered that the 'hail-shooting' campaign in Europe was made the subject of a conference held at Graz in 1902 . This body concluded that the results up to that time had been negative, and the hope was expressed that the experiments might be continued and carefully watched. The Italian Minister of Agriculture appointed a commission to undertake new experiments, under the presidency of Senator Blaserna. After a study of the results during the period 19021906 the conclusion reached by the Commission (Att. dei Lincei, 1906, II.; Ciel et Terre, January 16, 1907, 591-592) is that the fiveyear period has yielded an absolutely negative result. The commission reports that there is no hope of preventing damage by hail by means of the so-called 'hail-shooting;' and that protection must be sought along wholly different lines. The question may now be regarded as definitely settled.

\section{TROPICAL CYCLONE TRACKS}

We had occasion recently to call attention in these columns to a report by A. Schück, entitled 'Zur Kenntniss der Wirbelstürme' (Hamburg, 1905). A second report on the same subject has since been issued (dated 1906), presenting, in elaborate detail, the facts now at hand regarding the tracks of tropical cyclones in the West Indies, the Indian Ocean, and in the Pacific. Dr. Schück has made search through all available literature, and has plotted the tracks so far as known, on numerous charts. In future all students of cyclonology will need to refer to Schück's work. 